\title{
The effect of serum uric acid levels on the long-term prognosis of patients with non-ST-elevation myocardial infarction
}

\author{
Łukasz Kuźmaa,A-F, Agata Kulikowska ${ }^{2, B-D}$, Anna Kurasz ${ }^{1, B-D}$, Marta Maria Niwińska ${ }^{1, B-D}$, \\ Małgorzata Zalewska-Adamiec ${ }^{1, C, E, F}$, Sławomir Dobrzycki1, ${ }^{1, E, F}$, Hanna Bachórzewska-Gajewska ${ }^{1,3, A-F}$ \\ ${ }^{1}$ Department of Invasive Cardiology, Medical University of Bialystok, Poland \\ 2 Department of Anesthesiology and Intensive Therapy, Medical University of Bialystok, Poland \\ ${ }^{3}$ Department of Clinical Medicine, Medical University of Bialystok, Poland \\ A - research concept and design; B - collection and/or assembly of data; $\mathrm{C}$ - data analysis and interpretation; \\ $D$ - writing the article; $E$ - critical revision of the article; $F$ - final approval of the article
}

Address for correspondence

Łukasz Kuźma

E-mail:kuzma.lukasz@gmail.com

Funding sources

None declared

Conflict of interest

None declared

Received on April 30, 2020

Reviewed on August 6, 2020

Accepted on September 1, 2020

Cite as

Kuźma Ł, Kulikowska A, Kurasz A, et al. The effect of serum uric acid levels on the long-term prognosis of patients with non-ST-elevation myocardial infarction. Adv Clin Exp Med. 2020;29(11):1255-1263. doi:10.17219/acem/127145

DOI

10.17219/acem/127145

Copyright

Copyright by Author(s)

This is an article distributed under the terms of the

Creative Commons Attribution 3.0 Unported (CC BY 3.0)

(https://creativecommons.org/licenses/by/3.0/)

\begin{abstract}
Background. Cardiovascular diseases (CVD) are the leading cause of death worldwide. Many studies have reported an association between serum uric acid (SUA) and CVD, and its role as a risk marker for mortality.

Objectives. To assess the relationship between sUA levels and the long-term prognosis of patients with non-ST-elevation myocardial infarction (NSTEMI).

Material and methods. The study was conducted at the Clinical Hospital of Białystok (Poland). Based on medical records, 9328 patients were hospitalized between 2011 and 2013, of which 726 had NSTEMI. The exclusion criteria were: any diagnosed neoplasms and estimated glomerular filtration rate (eGFR) $<15 \mathrm{~mL} / \mathrm{min}$. The average observation time was 2324 days.

Results. A total of 549 patients were qualified for the analysis; men were in the majority $(69.03 \%, n=379)$, and the mean age was 68.42 years (standard deviation $(S D)=11.66)$. The sUA norm was exceeded in 137 of the patients (24.95\%). Hyperuricemia is more likely to occur in women (risk ratio (RR) $=1.52,95 \%$ confidence interval $(95 \% \mathrm{Cl})=1.016-2.288, \mathrm{p}=0.042)$, in patients with chronic kidney disease (CKD) $(\mathrm{RR}=3.452,95 \% \mathrm{Cl}=2.432-4.854, \mathrm{p}<0.001)$ and in patients with higher body mass index (BMI)s $(\mathrm{RR}=1.048,95 \% \mathrm{Cl}=1.008-1.090, \mathrm{p}=0.018)$. In the whole study group, during the study, 178 of the patients died (32.41.9\%), more often those with hyperuricemia: (60/137 patients (43.8\%)) compared to patients with normal sUA values (118/412 values (28.64\%)); $p<0.001)$. There was a significant correlation between an increase in sUA levels and an increase in mortality $(p<0.001)$. Independent risk factors of death were age $(R R=1.086,95 \% C l=1.065-1.108, p<0.001)$, SUA $(R R=1.245,95 \% C l=1.131-1.370, p=0.041)$, ejection fraction (EF) ( $\mathrm{RR}=0.928,95 \% \mathrm{Cl}=0.910-0.946, \mathrm{p}<0.001)$ and hemoglobin concentration $(\mathrm{RR}=0.685,95 \% \mathrm{Cl}=0.611-0.768, \mathrm{p}<0.001)$.
\end{abstract}

Conclusions. Serum UA concentration is an independent risk factor of long-term mortality in patients who have undergone NSTEMI, and is associated with higher in-hospital death rates. Secondary prevention after NSTEMI should entail management of the patients' sUA levels.

Key words: uric acid, myocardial infarction, mortality, hyperuricemia, gout 


\section{Introduction}

Cardiovascular disease (CVD) is the leading cause of death worldwide. ${ }^{1}$ Among other conditions, this group of diseases includes acute coronary syndromes (ACS). Their occurrence depends on risk factors that can be divided into modifiable (such as hypertension, diabetes, obesity, dyslipidemia, lack of physical activity, current smoking, moderate alcohol use) and non-modifiable (which include age, gender and family history). ${ }^{2}$ In order to prevent the occurrence of ACS more effectively, it is important to identify new modifiable risk factors as well as ways to control them.

The effects of excessive serum uric acid levels (sUA) were an intensely studied subject in recent decades. Although the physiopathological mechanisms leading to an increased risk of CVD are still a matter of debate, numerous studies have shown that uric acid (UA) is significantly associated with essential hypertension, ACS and chronic coronary syndrome, and heart failure, as well as with stroke, chronic kidney disease (CKD) and type 2 diabetes mellitus (DM2). Many researchers indicate that sUA is an independent risk factor for these pathologies. ${ }^{3-5}$

The prognostic value of sUA levels after ACS has also been investigated in several studies in recent years, and high levels of sUA on admission were found to be independently associated with in-hospital and long-term adverse outcomes across the whole spectrum of patients with ACS, including non-ST-segment elevation myocardial infarction (NSTEMI). ${ }^{6-13}$

Due to gradual increases in NSTEMI cases over STEMI and the limited number of studies focused on NSTEMI, we decided to investigate the relationship between sUA levels and the long-term prognosis of patients with NSTEMI in the study population. In addition, our work is novel in presenting very accurate clinical, biochemical, echocardiographic, and angiographic characteristics, which are not included in previous studies. The very long observation time, during which not only total mortality was assessed but also the type of revascularization treatment during the observation period, is also noteworthy.

The aim of the study was to assess the relationship between sUA levels and the long-term prognosis of patients with NSTEMI.

\section{Material and methods}

The study was conducted at the Clinical Hospital of the Medical University of Bialystok (Poland). Based on the medical records, 9328 patients were hospitalized (1594 with ACS) between 2011 and 2013, of which 726 were NSTEMI cases.

From the group of patients with NSTEMI, our analysis excluded 177 patients with diagnosed neoplasms, with estimated glomerular filtration rate (eGFR) $<15$, those lost in follow-up and those with missing data, leaving $549 \mathrm{pa}-$ tients in the final analysis.

The norm for UA concentration was defined as $6 \mathrm{mg} / \mathrm{dL}$ for women and $7 \mathrm{mg} / \mathrm{dL}$ for men, as is standard in most studies. ${ }^{13}$ The norm was exceeded by 52 women $(30.6 \%)$ and 85 men (22.4\%). The study population was divided into quartiles on the basis of their sUA levels, and for each of them, a comparative analysis and long-term observation was conducted.

\section{Long-term observation}

We conducted a two-stage follow-up examination. In the $1^{\text {st }}$ stage, the general type of planned revascularization treatment was evaluated, and in the $2^{\text {nd }}$ stage, we investigated total mortality. All-cause mortality was collected from the PESEL registry of the Polish Ministry of Digital Affairs on November 14, 2018.

The average time from inclusion in the study to death or the end of observation was 1866 days (standard deviation $(\mathrm{SD})=840$ ); the median was 2092 days. In the group of living patients, the average observation time was 2324 days (SD = 327); the median was 2335 days. In the whole study group, 178 people died (32.4\%). In the group of deceased patients, the average follow-up time was 913 days ( $\mathrm{SD}=791)$; the median was 660 days.

\section{Statistical analysis}

In the statistical analysis, the distribution of variables was evaluated using the Kolmogorov-Smirnov test. The two-tailed T-test and analysis of variance (ANOVA) test were used for comparative analysis. Non-normally distributed data was compared using the Mann-Whitney test. The results obtained were presented as mean values with $\mathrm{SD}$ or as percentage values corresponding to relative frequency.

Pearson's rank correlation test was applied for evaluating relationships among the biochemical parameters. A forward stepwise (Wald) logistic regression was used to determine mortality risk factors. The results were presented as risk ratios (RR) from the $5^{\text {th }}$ to the $95^{\text {th }}$. A two-sided p-value $\leq 0.05$ was considered significant. All analyses were performed using MS Excel v. 16.40 (Microsoft Corp., Redmond, USA) and SPSS IBM software v. 26 (IBM Corp., Armonk, USA).

The study was approved by the Bioethics Committee of the Medical University of Bialystok (approval No. R-1-002/18/2019).

\section{Results}

A total of 549 patients were qualified for the analysis, with men in the majority ( $\mathrm{n}=379,69.03 \%)$. The mean age of the entire group was 68.42 years $(\mathrm{SD}=11.66)$. The majority of the patients $(n=422,76.87 \%)$ were burdened with 
Table 1. Characteristics of the studied population

\begin{tabular}{|c|c|}
\hline Parameter & $\begin{array}{l}\text { Studied population } \\
\qquad(\mathrm{n}=549)\end{array}$ \\
\hline Age [years], mean (SD) & $68.42(11.66)$ \\
\hline Male, \% (n) & $69.03(379)$ \\
\hline BMI $\left[\mathrm{kg} / \mathrm{m}^{2}\right]$, mean (SD) & $27.81(4.92)$ \\
\hline SBP on admission [mm Hg], mean (SD) & $139.7(23.46)$ \\
\hline DBP on admission [mm Hg], mean (SD) & $79.81(13.71)$ \\
\hline Heart rate on admission [mm Hg], mean (SD) & $77.42(17.61)$ \\
\hline $\mathrm{EF}[\%]$, mean $(\mathrm{SD})$ & $44.36(10.68)$ \\
\hline Gout, \% (n) & $4.19(23)$ \\
\hline Arterial hypertension, \% (n) & $76.87(422)$ \\
\hline $\mathrm{DM} 2, \%(\mathrm{n})$ & $25.50(140)$ \\
\hline Previous myocardial infarction, \% (n) & $22.40(123)$ \\
\hline$C K D, \%(n)$ & $28.78(158)$ \\
\hline sUA concentration $[\mathrm{mg} / \mathrm{dL}]$, mean (SD) & $5.78(1.89)$ \\
\hline Exceeded sUA norm, \% (n) & $24.95(137)$ \\
\hline $\begin{array}{l}\text { Serum creatinine concentration }[\mathrm{mg} / \mathrm{dL}] \text {, mean } \\
\text { (SD) }\end{array}$ & $1.00(0.35)$ \\
\hline eGFR $\left[\mathrm{mL} / \mathrm{min} / 1.73 \mathrm{~m}^{2}\right]$, mean (SD) & $75.67(21.99)$ \\
\hline Hemoglobin concentration [g/dL], mean (SD) & $13.69(1.79)$ \\
\hline Platelet count $\left[\times 10^{3} / \mathrm{mm}^{3}\right]$, mean (SD) & $221.08(88.25)$ \\
\hline Leukocytes count $\left[\times 10^{6} / \mathrm{mm}^{3}\right]$, mean (SD) & $9.23(5.08)$ \\
\hline $\begin{array}{l}\text { Total serum cholesterol concentration }[\mathrm{mg} / \mathrm{dL}] \\
\text { mean }(\mathrm{SD})\end{array}$ & $198.04(51.79)$ \\
\hline $\begin{array}{l}\text { Serum LDL cholesterol concentration }[\mathrm{mg} / \mathrm{dL}] \text {, } \\
\text { mean (SD) }\end{array}$ & $130.49(47.65)$ \\
\hline $\begin{array}{l}\text { Serum HDL cholesterol concentration }[\mathrm{mg} / \mathrm{dL}] \text {, } \\
\text { mean }(\mathrm{SD})\end{array}$ & $47.67(12.53)$ \\
\hline $\begin{array}{l}\text { Serum triglyceride concentration }[\mathrm{mg} / \mathrm{dL}] \text {, } \\
\text { mean (SD) }\end{array}$ & $120.32(84.88)$ \\
\hline $\begin{array}{l}\text { Serum glucose concentration on admission } \\
{[\mathrm{mg} / \mathrm{dL}] \text {, mean (SD) }}\end{array}$ & $128.71(45.84)$ \\
\hline Potassium concentration [mEq/L], mean (SD) & $4.28(0.43)$ \\
\hline Sodium concentration [mEq/L], mean (SD) & $138.92(3.52)$ \\
\hline
\end{tabular}

arterial hypertension; 158 (28.78\%) had CKD; 140 (25.5\%) had DM2; 123 (22.4\%) had a history of previous myocardial infarction; and 23 (4.19\%) suffered from gout. All the patients had undergone invasive treatments. Nearly 1 in 3 patients had a narrowing of more than 1 coronary artery. The most common procedure they had undergone was a percutaneous coronary intervention (PCI) on the left anterior descending coronary artery (LAD; $\mathrm{n}=156,28.42 \%$;). Coronary artery bypass surgery (CABG) had been performed on 34 (6.19\%) of the patients. During the followup period, 168 (30.06\%) of the patients had a PCI and 50 (9.11\%) underwent a CABG procedure (Table 1 ).

The mean concentration of sUA in the study group was $5.78 \mathrm{mg} / \mathrm{dL}(\mathrm{SD}=1.89 \mathrm{mg} / \mathrm{dL})$. The lowest observed value was $1.92 \mathrm{mg} / \mathrm{dL}$. In 133 (25\%) of the patients, the sUA concentration was below $4.42 \mathrm{mg} / \mathrm{dL}$, and in 149 (25\%) of the group the level was above $6.69 \mathrm{mg} / \mathrm{dL}$. The highest observed value

\begin{tabular}{|c|c|}
\hline Parameter & $\begin{array}{l}\text { Studied population } \\
\qquad(\mathrm{n}=549)\end{array}$ \\
\hline Fibrinogen concentration $[\mathrm{mg} / \mathrm{dL}]$, mean (SD) & $432.50(107.86)$ \\
\hline Patients with eGFR $>90 \mathrm{~mL} / \mathrm{min} / 73 \mathrm{~m}^{2}, \%$ (n) & $31.33(172)$ \\
\hline $\begin{array}{l}\text { Patients with eGFR } 60-90 \mathrm{~mL} / \mathrm{min} / 1.73 \mathrm{~m}^{2} \text {, } \\
\%(\mathrm{n})\end{array}$ & $44.26(243)$ \\
\hline $\begin{array}{l}\text { Patients with eGFR } 45-60 \mathrm{~mL} / \mathrm{min} / 1.73 \mathrm{~m}^{2} \text {, } \\
\%(\mathrm{n})\end{array}$ & $12.75(70)$ \\
\hline $\begin{array}{l}\text { Patients with eGFR } 30-45 \mathrm{~mL} / \mathrm{min} / 1.73 \mathrm{~m}^{2} \text {, } \\
\%(\mathrm{n})\end{array}$ & $8.38(46)$ \\
\hline $\begin{array}{l}\text { Patients with eGFR } 15-30 \mathrm{~mL} / \mathrm{min} / 1.73 \mathrm{~m}^{2} \text {, } \\
\%(\mathrm{n})\end{array}$ & $3.28(18)$ \\
\hline Multi vessel disease & $37.83(199)$ \\
\hline $\mathrm{PCI} L M, \%(\mathrm{n})$ & $4.19(23)$ \\
\hline$P C I L A D, \%(n)$ & $28.42(156)$ \\
\hline $\mathrm{PCI}$ RCA, \% (n) & $24.77(136)$ \\
\hline PCI Diag, \% (n) & $4.74(26)$ \\
\hline $\mathrm{PCl} C \times, \%(n)$ & $25.14(138)$ \\
\hline PCI IM, \% (n) & $2.01(11)$ \\
\hline $\mathrm{PCI} M, \%(n)$ & $11.11(61)$ \\
\hline CABG, $\%(n)$ & $6.19(34)$ \\
\hline In-hospital mortality, \% (n) & $2.55(14)$ \\
\hline Conservative treatment during follow-up, \% (n) & $51.55(283)$ \\
\hline CABG during follow-up, \% (n) & $9.11(50)$ \\
\hline $\mathrm{PCl}$ during follow-up, \% (n) & $30.6(168)$ \\
\hline Deaths during follow-up, \% (n) & $32.42(178)$ \\
\hline
\end{tabular}

BMI - body mass index; SBP - systolic blood pressure; sUA - serum uric acid; DBP - diastolic blood pressure; DM2 - diabetes mellitus type 2; CABG - coronary artery bypass grafting; Cx - circumflex artery; EF - ejection fraction; CKD - chronic kidney disease; eGFR - estimated glomerular filtration rate; $\mathrm{HDL}$ - high density lipoprotein; IM - intermediate; LDL - low density lipoprotein; LMCA - left main coronary artery; LAD - left anterior descending artery; $M$ - marginal; $\mathrm{PCl}$ - percutaneous coronary intervention; PDA - posterior descending artery; RCA - right coronary artery; SD - standard deviation.

was $14.12 \mathrm{mg} / \mathrm{dL}$. The median sUA value was $5.42 \mathrm{mg} / \mathrm{dL}$ (interquartile range $(\mathrm{IQR})=2.27$ ) (Table 2, Fig. 1).

Serum UA concentration correlates inversely with total serum cholesterol concentration $(\mathrm{R}=-0.10)$ and serum high-density-lipoprotein (HDL) cholesterol concentration $(R=-0.26)$. However, there is a positive correlation between sUA and creatinine concentration $(\mathrm{R}=0.50)$, and between sUA and triglyceride $(\mathrm{R}=0.14)$ concentration (Table 3$)$.

The group with elevated sUA values (over $6.69 \mathrm{mg} / \mathrm{dL}$ ) was significantly older than the one with sUA values under $4.42 \mathrm{mg} / \mathrm{dL}: 72.30$ (SD = 10.38) compared to 67.13 $(\mathrm{SD}=11.78) ; \mathrm{p}<0.001$. The elevated group also had a significantly higher heart rate on admission (81.9 (SD = 18.09) compared to $75.93(\mathrm{SD}=17.21)$ ); $\mathrm{p}<0.001)$ and a lower ejection fraction $(\mathrm{EF})(41.11(\mathrm{SD}=12.16)$ compared to 45.44 $(\mathrm{SD}=9.93) ; \mathrm{p}<0.001)$ than the group with sUA values under $4.42 \mathrm{mg} / \mathrm{dL}$. Additionally, gout was diagnosed more 
Table 2. Serum uric acid concentration in the study population

\begin{tabular}{|l|c|}
\multicolumn{1}{|c|}{ Parameter } & UA [mg/dL] \\
\hline Minimum & 1.92 \\
\hline Maximum & 14.12 \\
\hline Mean & 5.78 \\
\hline SD & 1.89 \\
\hline Median & 5.42 \\
\hline IQ & 4.42 \\
\hline 3Q & 6.69 \\
\hline IQR & 2.27 \\
\hline Sum & 549 \\
\hline
\end{tabular}

UA - uric acid; IQR - interquartile range; SD - standard deviation; $1 \mathrm{Q}$ - $1^{\text {st }}$ quartile; $3 Q-3^{\text {rd }}$ quartile.

Table 3. Pearson's correlations between biochemical parameters (significant results were underlined, $\mathrm{p}<0.05$ )

\begin{tabular}{|l|c|}
\hline \multicolumn{1}{|c|}{ Parameter } & $\mathrm{R}$ \\
\hline Creatinine concentration & 0.50 \\
\hline Fibrinogen concentration & 0.02 \\
\hline Hemoglobin concentration & 0.02 \\
\hline Platelet count & -0.08 \\
\hline Leukocytes & 0.04 \\
\hline Total serum cholesterol concentration & -0.10 \\
\hline Serum LDL cholesterol concentration & -0.07 \\
\hline Serum HDL cholesterol concentration & -0.26 \\
\hline Serum triglyceride concentration & 0.14 \\
\hline Serum glucose concentration & 0.09 \\
\hline Potassium concentration & 0.10 \\
\hline Sodium concentration & -0.07 \\
\hline
\end{tabular}

LDL - low-density lipoprotein; HDL - high-density lipoprotein.

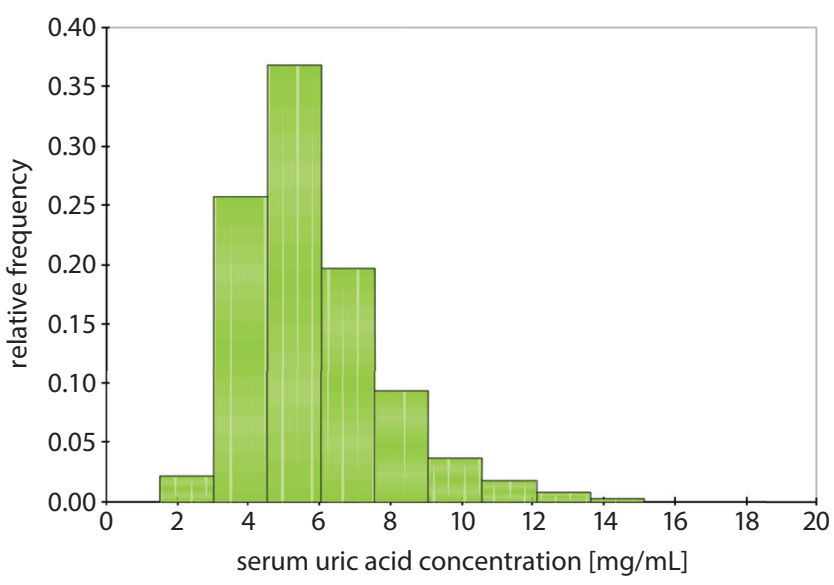

Fig. 1. Histogram of sUA concentration

frequently in this group (16 patients (11.67\%) compared to 7 patients $(1.70 \%) ; \mathrm{p}<0.001)$ ), as well as CKD (84 patients $(61.31 \%)$ compared to 74 patients $(17.96 \%) ; \mathrm{p}<0.001)$, which was associated with differences in the frequency of different ranges of eGFR values. We also observed that the group with elevated sUA values had a significantly higher average

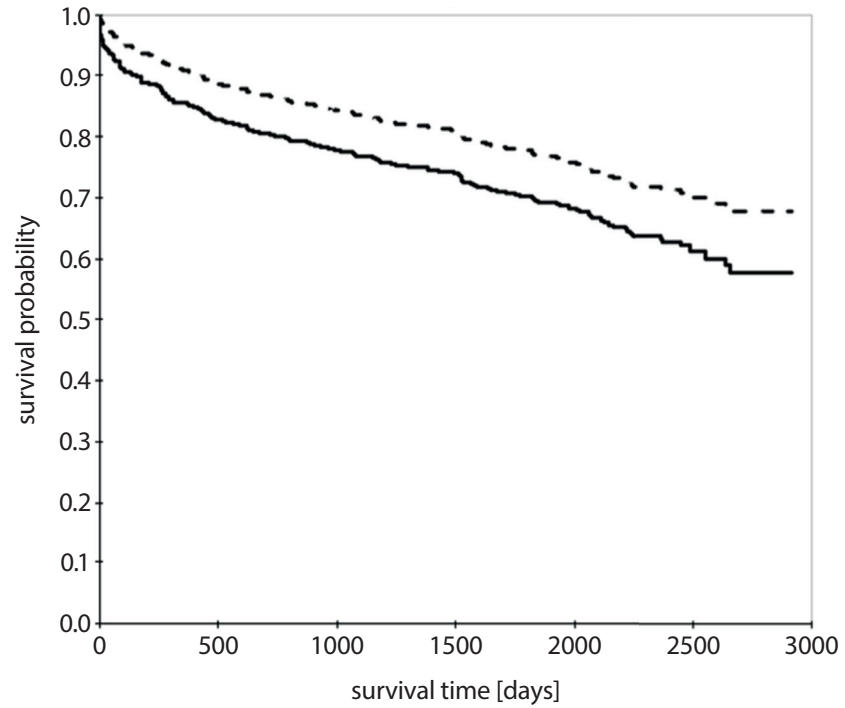

Fig. 2. Kaplan-Meier survival curve. Survival curves for normal serum uric acid values (continuous line) vs exceeded serum uric acid values (dashed line).

serum creatinine concentration $(1.25 \mathrm{mg} / \mathrm{dL}(\mathrm{SD}=0.46)$ compared to $0.91 \mathrm{mg} / \mathrm{dL}(\mathrm{SD}=0.26) ; \mathrm{p}<0.001)$; a lower average eGFR $\left(57.92 \mathrm{~mL} / \mathrm{min} / 1.73 \mathrm{~m}^{2}(\mathrm{SD}=22.65) \mathrm{com}\right.$ pared to $\left.81.58 \mathrm{~mL} / \mathrm{min} / 1.73 \mathrm{~m}^{2}(\mathrm{SD}=18.31) ; \mathrm{p}<0.001\right)$; and a lower average serum HDL cholesterol concentration $(43.68 \mathrm{mg} / \mathrm{dL}(\mathrm{SD}=13.43)$ compared to $48.98 \mathrm{mg} / \mathrm{dL}$ $(\mathrm{SD}=11.95) ; \mathrm{p}<0.001)$. Additionally, patients with hyperuricemia died more often during the study (60/137 patients $(43.8 \%)$ ) compared to patients with normal sUA values $(118 / 412$ values $(28.64 \%))$; $\mathrm{p}=0.01)$ and had a higher in-hospital death rate $(8 / 137$ patients $(5.84 \%)$ compared to $6 / 412$ patients $(1.46 \%) ; \mathrm{p}=0.04$ ) (Table 4 , Fig. 2 ).

A multivariable regression analysis showed that hyperuricemia was more likely to occur in women $(R R=1.52$, $95 \%$ confidence interval $(95 \% \mathrm{CI})=1.016-2.288, \mathrm{p}=0.042)$ and in patients with CKD $(\mathrm{RR}=3.452,95 \% \mathrm{CI}=2.432-$ 4.854, $\mathrm{p}<0.001)$. People with higher body mass index $(\mathrm{BMI})(\mathrm{RR}=1.048,95 \% \mathrm{CI}=1.008-1.090, \mathrm{p}=0.018)$ were more likely to have increased sUA levels, but this likelihood decreased with an increase in EF $(R R=0.964$, 95\% CI $=0.946-0.981, \mathrm{p}<0.001)$ or in HDL concentration $(\mathrm{RR}=0.963,95 \% \mathrm{CI}=0.946-0.980, \mathrm{p}<0.001)$ (Table 5).

The study population was divided into quartiles based on their sUA level. Patients in the $4^{\text {th }}$ quartile (4Q) were the oldest ( $\mathrm{p}<0.001)$ and showed the lowest EF ( $\mathrm{p}<0.001)$. The $2^{\text {nd }}$ quartile (2Q) was associated with the rarest occurrence of DM2 and hypertension $(\mathrm{p}<0.001)$. With increasing concentrations of sUA, the occurrence of CKD and death increased ( $<<0.001$ ), especially in the 4Q (Table 6, Fig. 3).

The patients who died during the observation period were statistically older than those who survived (74.76 years $(S D=9.86)$ compared to 65.37 years $(\mathrm{SD}=11.23)$; $\mathrm{p}$ < 0.001); had higher heart rates on admission $(82.53 \mathrm{bpm}(\mathrm{SD}=18.61)$ compared to $74.97 \mathrm{bpm}$ $(\mathrm{SD}=16.59, \mathrm{p}<0.001)$; had lower EF $(38.84 \%(\mathrm{SD}=12.21)$ 
Table 4. Comparison of patients with exceeded sUA value and normal

\begin{tabular}{|c|c|c|c|}
\hline Parameter & $\begin{array}{l}\text { Exceeded value } \\
\qquad(n=137)\end{array}$ & Normal value $(n=412)$ & $\mathrm{p}$-value \\
\hline Age [years], mean (SD) & $72.30(10.38)$ & $67.13(11.78)$ & $<0.001$ \\
\hline Male, \% (n) & $62.04(85)$ & $71.36(294)$ & 0.045 \\
\hline BMI [kg/m²], mean (SD) & $28.68(5.33)$ & $27.52(4.75)$ & 0.03 \\
\hline SBP on admission [mm Hg], mean (SD) & $137.56(23.37)$ & $140.42(23.47)$ & 0.22 \\
\hline DBP on admission [mm Hg], mean (SD) & $78.86(14.82)$ & $80.13(13.32)$ & 0.37 \\
\hline Heart rate on admission [mm Hg], mean (SD) & $81.9(18.09)$ & $75.93(17.21)$ & $<0.001$ \\
\hline $\mathrm{EF}[\%]$, mean (SD) & $41.11(12.16)$ & $45.44(9.93)$ & $<0.001$ \\
\hline Arterial hypertension, \% (n) & $81.02(111)$ & $75.49(311)$ & 0.16 \\
\hline $\mathrm{DM} 2, \%(\mathrm{n})$ & $35.77(49)$ & $22.09(91)$ & 0.003 \\
\hline Gout, \% (n) & $11.67(16)$ & $1.7(7)$ & $<0.001$ \\
\hline Previous myocardial infarction, \% (n) & $32.12(44)$ & $19.17(79)$ & 0.004 \\
\hline$C K D, \%(n)$ & $61.31(84)$ & $17.96(74)$ & $<0.001$ \\
\hline Patients with eGFR $\geq 90 \mathrm{~mL} / \mathrm{min} / 1.73 \mathrm{~m}^{2}, \%$ (n) & $8.76(12)$ & $38.83(160)$ & $<0.001$ \\
\hline Patients with eGFR 60-89 mL/min/1.73 m²,\% (n) & $35.04(48)$ & $47.33(195)$ & 0.01 \\
\hline Patients with eGFR $45-59 \mathrm{~mL} / \mathrm{min} / 1.73 \mathrm{~m}^{2}, \%$ (n) & $23.36(32)$ & $9.22(38)$ & $<0.001$ \\
\hline Patients with eGFR 30-44 mL/min/1.73 m²,\% (n) & $23.36(32)$ & $3.4(14)$ & $<0.001$ \\
\hline Patients with eGFR 15-29 mL/min/1.73 m², \% (n) & $9.49(13)$ & $1.21(5)$ & 0.002 \\
\hline PCI LM [\%] (n) & $3.65(5)$ & $4.37(18)$ & 0.71 \\
\hline PCI LAD [\%] (n) & $32.85(45)$ & $26.94(111)$ & 0.20 \\
\hline PCI RCA [\%] (n) & $27.01(37)$ & $24.03(99)$ & 0.49 \\
\hline PCI Diag [\%] (n) & $2.19(3)$ & $5.58(23)$ & 0.045 \\
\hline $\mathrm{PCl} \times x[\%](n)$ & $22.63(31)$ & $25.97(107)$ & 0.43 \\
\hline $\mathrm{PCl} \operatorname{IM}[\%](n)$ & $0.73(1)$ & $2.43(10)$ & 0.11 \\
\hline $\mathrm{PCI} M[\%](n)$ & $10.95(15)$ & $11.17(46)$ & 0.94 \\
\hline Further conservative treatment, \% (n) & $46.72(64)$ & $53.16(219)$ & 0.19 \\
\hline CABG during admission, \% (n) & $6.57(9)$ & $6.07(25)$ & 0.84 \\
\hline Elective CABG, \% (n) & $12.41(17)$ & $8.01(33)$ & 0.16 \\
\hline Elective PCl, \% (n) & $28.47(39)$ & $31.31(129)$ & 0.53 \\
\hline In-hospital mortality, \% (n) & $5.84(8)$ & $1.46(6)$ & 0.04 \\
\hline Deaths, \% (n) & $43.8(60)$ & $28.64(118)$ & $<0.001$ \\
\hline Serum creatinine concentration [mg/dL], mean (SD) & $1.25(0.46)$ & $0.91(0.26)$ & $<0.001$ \\
\hline eGFR, CKD-EPI (mL/min/1.73 m²,\% (n)), mean (SD) & $57.92(22.65)$ & $81.58(18.31)$ & $<0.001$ \\
\hline Hemoglobin concentration [g/dL], mean (SD) & $13.37(2.05)$ & $13.79(1.68)$ & 0.03 \\
\hline Platelet count $\left[\times 10^{3} / \mathrm{mm}^{3}\right]$, mean (SD) & $219.08(110.74)$ & $221.75(79.53)$ & 0.80 \\
\hline Leukocytes $\left[\times 10^{6} / \mathrm{mm}^{3}\right]$, mean (SD) & $9.44(1.29)$ & $9.16(3.78)$ & 0.42 \\
\hline Total serum cholesterol concentration [mg/dL], mean (SD) & $186.41(55.9)$ & $201.92(49.82)$ & 0.005 \\
\hline Serum LDL cholesterol concentration [mg/dL], mean (SD) & $119.73(48.8)$ & $134.08(46.78)$ & 0.003 \\
\hline Serum HDL cholesterol concentration [mg/dL], mean (SD) & $43.68(13.43)$ & $48.98(11.95)$ & $<0.001$ \\
\hline Serum triglyceride concentration [mg/dL], mean (SD) & $141.27(107.59)$ & $113.34(74.68)$ & 0.006 \\
\hline Serum glucose concentration on admission [mg/dL], mean (SD) & $139.43(49.36)$ & $125.14(44.1)$ & 0.003 \\
\hline Potassium concentration [mEq/L], mean (SD) & $4.36(0.52)$ & $4.25(0.4)$ & 0.03 \\
\hline Sodium concentration [mEq/L], mean (SD) & $138.41(4.5)$ & $139.09(3.11)$ & 0.10 \\
\hline Fibrinogen concentration [mg/dL], mean (SD) & $445.56(110.77)$ & $13.79(1.68)$ & 0.11 \\
\hline
\end{tabular}

$\mathrm{BMI}$ - body mass index; SBP - systolic blood pressure; sUA - serum uric acid; DBP - diastolic blood pressure; DM2 - diabetes mellitus type 2;

CABG - coronary artery bypass grafting; Cx - circumflex artery; EF - ejection fraction; CKD - chronic kidney disease; eGFR - estimated glomerular filtration rate; CKD-EPI - chronic kidney disease-epidemiology collaboration formula; HDL - high density lipoprotein; IM - intermediate; LDL - Iow density lipoprotein; LMCA - left main coronary artery; LAD - left anterior descending artery; $\mathrm{M}$ - marginal; $\mathrm{PCl}$ - percutaneous coronary intervention; PDA - posterior descending artery; RCA - right coronary artery; SD - standard deviation. 
Table 5. Multivariable logistic regression forward stepwise Wald method - OR for exceeding the norm of UA concentration

\begin{tabular}{|c|c|c|c|}
\hline Parameter & $p$-value & $\mathrm{RR}$ & $95 \% \mathrm{Cl}$ for $\mathrm{RR}$ \\
\hline Male & 0.042 & 0.656 & $0.437-0.984$ \\
\hline Age (for every 1 year) & $<0.001$ & 1.042 & $1.023-1.060$ \\
\hline CKD & $<0.001$ & 3.452 & $2.432-4.854$ \\
\hline EF (for each $1 \%$ increase) & $<0.001$ & 0.964 & $0.946-0.981$ \\
\hline BMI (for each 1 kg/m² increase) & 0.018 & 1.048 & $1.008-1.090$ \\
\hline HDL (for each 1 mg/dL increase) & $<0.001$ & 0.963 & $0.946-0.980$ \\
\hline
\end{tabular}

$\mathrm{R}^{2}$ Nagelkerke - 0.89; BMI - body mass index; Cl - confidence interval; EF - ejection fraction; HDL - high density lipoprotein; RR - risk ratio; OR - odds ratio; UA - uric acid; CKD - chronic kidney disease.

Table 6. Quartiles of sUA in the study population

\begin{tabular}{|c|c|c|c|c|c|}
\hline Parameters Quartiles & $\begin{array}{c}1 \mathrm{Q} \\
(<4.42) \\
(\mathrm{n}=133)\end{array}$ & $\begin{array}{c}2 \mathrm{Q} \\
(4.42-5.42) \\
(\mathrm{n}=132)\end{array}$ & $\begin{array}{c}3 Q \\
(5.36-6.69) \\
(n=135)\end{array}$ & $\begin{array}{c}4 Q \\
(>6.69) \\
(n=149)\end{array}$ & $\mathrm{p}$-value \\
\hline Male, \% (n) & $57.14(76)$ & $68.18(90)$ & $72.59(98)$ & $77.18(115)$ & $p<0.001$ \\
\hline Age [years], mean, (SD) & $67.56(11.46)$ & $66.71(11.89)$ & $68.15(12.7)$ & $70.93(10.24)$ & $p<0.001$ \\
\hline EF (\%), mean, (SD) & $45.32(9.29)$ & $46.09(10.26)$ & $45.96(9.05)$ & $40.53(12.57)$ & $p<0.001$ \\
\hline Hypertension, \%, (n) & $77.44(103)$ & $69.7(92)$ & $79.26(107)$ & $80.54(120)$ & $p<0.001$ \\
\hline $\mathrm{DM} 2, \%,(\mathrm{n})$ & $26.32(35)$ & $18.94(25)$ & $23.7(32)$ & $32.21(48)$ & $p<0.001$ \\
\hline$C K D, \%,(n)$ & $12.78(17)$ & $18.18(24)$ & $25.19(34)$ & $55.7(83)$ & $p<0.001$ \\
\hline Deaths, \%, (n) & $27.82(37)$ & $28.03(37)$ & $27.41(37)$ & $44.97(67)$ & $p<0.001$ \\
\hline
\end{tabular}

CKD - chronic kidney disease; EF - ejection fraction; DM2 - type 2 diabetes mellitus; SD - standard deviation; $1 Q$ - $1^{\text {st }}$ quartile; $2 Q$ - $2^{\text {nd }}$ quartile; $3 Q$ - $3^{\text {rd }}$ quartile; $4 \mathrm{Q}-4^{\text {th }}$ quartile.

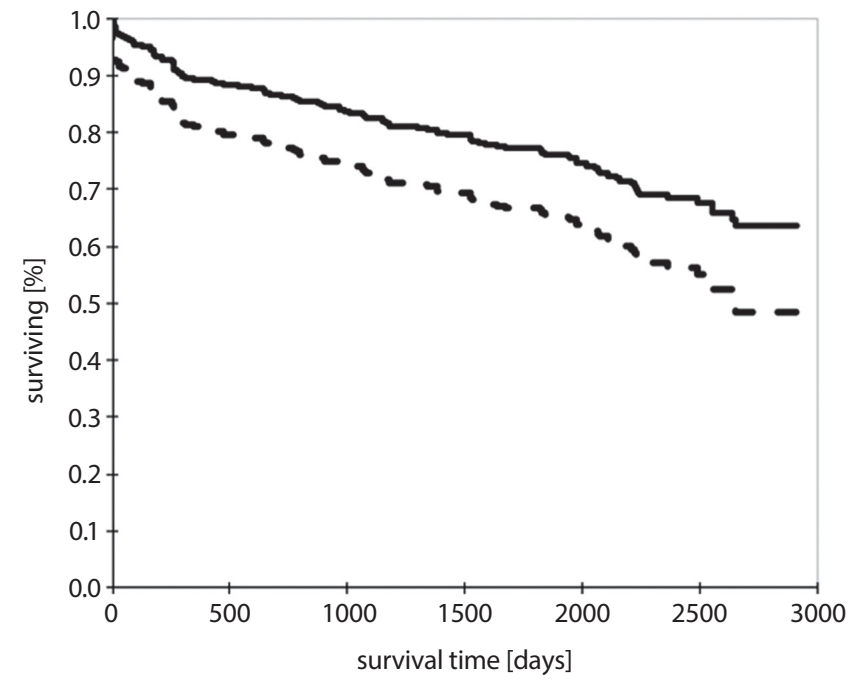

Fig. 3. Kaplan-Meier survival curve. Survival curves for 1 quartile of serum uric acid concentration (continuous line) vs 4 quartile (dashed line)

compared to $46.96 \%$ (SD = 8.77); $\mathrm{p}<0.001)$; and more frequently had a history of DM2 (59 (33.15\%) compared to 81 (21.83\%); $\mathrm{p}=0.007)$ and myocardial infarction (54 (30.34\%) compared to 69 (18.6\%); $\mathrm{p}=0.004)$. They were diagnosed more frequently with CKD (83 patients $(46.63 \%)$ compared to 75 (20.22\%); $\mathrm{p}<0.001)$; therefore, they were predominantly in the eGFR ranges of $45-59 \mathrm{~mL} / \mathrm{min}$ and $30-$ $44 \mathrm{~mL} / \mathrm{min}$. Regarding invasive artery treatment, the only difference between the group who died and the survivors was that PCIs for the left main coronary artery (PCI LM) had been performed more often on the patients who died (14 $(7.87 \%)$ compared to $9(2.43 \%)$; $\mathrm{p}=0.013)$. The patients who died also had higher concentrations of sUA and creatinine, along with lower eGFR and hemoglobin concentration. On admission, they had lower concentrations of total cholesterol $(185.3 \mathrm{mg} / \mathrm{dL}(\mathrm{SD}=52.76) \mathrm{com}-$ pared to $204.1 \mathrm{mg} / \mathrm{dL}(\mathrm{SD}=50.28) ; \mathrm{p}<0.001)$ and its fractions than the survivors; they also had higher concentrations of glucose $(138.81 \mathrm{mg} / \mathrm{dL}(\mathrm{SD}=52.49)$ compared to $123.85 \mathrm{mg} / \mathrm{dL}(\mathrm{SD}=41.5) ; \mathrm{p}<0.001)$ and fibrinogen $(466 \mathrm{mg} / \mathrm{mL}(\mathrm{SD}=115.52)$ compared to $416.43 \mathrm{mg} / \mathrm{mL}$ $(\mathrm{SD}=100.24) ; \mathrm{p}<0.001)$ (Table 7).

The multivariable regression analysis showed that the risk of death increased significantly with age $(R R=1.086$, $95 \% \mathrm{CI}=1.065-1.108, \mathrm{p}<0.001)$ and increases in sUA concentration $(\mathrm{RR}=1.245,95 \% \mathrm{CI}=1.131-1.370, \mathrm{p}=0.041)$, but also decreased with increases in EF $(R R=0.928$, 95\% CI $=0.910-0.946, \mathrm{p}<0.001)$ and hemoglobin concentrations $(\mathrm{RR}=0.685,95 \% \mathrm{CI}=0.611-0.768, \mathrm{p}<0.001)$ (Table 8).

\section{Discussion}

In this long-term observational study, we assessed the influence of sUA levels among patients with NSTEMI. We found that a high sUA level was a factor that increased mortality. The study also showed that high levels 
Table 7. Comparison of dead and surviving patients

\begin{tabular}{|c|c|c|c|}
\hline Parameter & Alive patients $(n=371)$ & Dead patients $(n=178)$ & $\mathrm{p}$-value \\
\hline Age [years], mean (SD) & $65.37(11.23)$ & $74.76(9.86)$ & $<0.001$ \\
\hline Male, \% (n) & $71.16(264)$ & $64.61(115)$ & 0.128 \\
\hline BMI $\left[\mathrm{kg} / \mathrm{m}^{2}\right]$, mean (SD) & $28.05(4.76)$ & $27.32(5.23)$ & 0.115 \\
\hline SBP on admission [mm Hg], mean (SD) & $141.15(22.22)$ & $136.7(25.64)$ & 0.048 \\
\hline DBP on admission [mm Hg], mean (SD) & $80.83(13.04)$ & $77.7(14.82)$ & 0.017 \\
\hline Heart rate on admission [mm Hg], mean (SD) & $74.97(16.59)$ & $82.53(18.61)$ & $<0.001$ \\
\hline $\mathrm{EF}[\%]$, mean (SD) & $46.96(8.77)$ & $38.84(12.21)$ & $<0.001$ \\
\hline Arterial hypertension, \% (n) & $76.01(282)$ & $78.65(140)$ & 0.487 \\
\hline $\mathrm{DM} 2, \%(\mathrm{n})$ & $21.83(81)$ & $33.15(59)$ & 0.007 \\
\hline Gout, \% (n) & $3.5(13)$ & $5.62(10)$ & 0.286 \\
\hline Previous myocardial infarction, \% (n) & $18.6(69)$ & $30.34(54)$ & 0.004 \\
\hline$C K D, \%(n)$ & $20.22(75)$ & $46.63(83)$ & $<0.001$ \\
\hline eGFR $\geq 90 \mathrm{~mL} / \mathrm{min} / 1.73 \mathrm{~m}^{2}, \%(\mathrm{n})$ & $40.16(149)$ & $12.92(23)$ & $<0.001$ \\
\hline eGFR 60-90 mL/min/1.73 m²,\% (n) & $43.94(163)$ & $44.94(80)$ & 0.824 \\
\hline eGFR $45-60 \mathrm{~mL} / \mathrm{min} / 1.73 \mathrm{~m}^{2}, \%(\mathrm{n})$ & $8.63(32)$ & $21.35(38)$ & $<0.001$ \\
\hline eGFR 30-45 mL/min/1.73 m²,\% (n) & $5.39(20)$ & $14.61(26)$ & 0.002 \\
\hline eGFR $15-30 \mathrm{~mL} / \mathrm{min} / 1.73 \mathrm{~m}^{2}, \%(\mathrm{n})$ & $1.89(7)$ & $6.18(11)$ & 0.028 \\
\hline PCI LM, \% (n) & $2.43(9)$ & $7.87(14)$ & 0.013 \\
\hline PCI LAD, \% (n) & $30.19(112)$ & $24.72(44)$ & 0.175 \\
\hline $\mathrm{PCI}$ RCA, \% (n) & $25.07(93)$ & $24.16(43)$ & 0.817 \\
\hline PCI Diag, \% (n) & $4.31(16)$ & $5.62(10)$ & 0.52 \\
\hline $\mathrm{PCl} C \mathrm{x}, \%(n)$ & $25.07(93)$ & $25.28(45)$ & 0.957 \\
\hline $\mathrm{PCI} I \mathrm{M}, \%(n)$ & $2.16(8)$ & $1.69(3)$ & 0.701 \\
\hline $\mathrm{PCl} M, \%(n)$ & $11.05(41)$ & $11.24(20)$ & 0.949 \\
\hline $\mathrm{CABG}, \%(\mathrm{n})$ & $53.37(198)$ & $47.75(85)$ & 0.219 \\
\hline Conservative treatment during follow-up, \% (n) & $6.2(23)$ & $6.18(11)$ & 0.993 \\
\hline CABG treatment during follow-up, \% (n) & $7.82(29)$ & $11.8(21)$ & 0.156 \\
\hline $\mathrm{PCl}$ treatment during follow-up, \% (n) & $32.61(121)$ & $26.4(47)$ & 0.132 \\
\hline sUA concentration [mg/dL], mean (SD) & $5.52(1.6)$ & $6.32(2.31)$ & $<0.001$ \\
\hline Exceeded sUA norm, \% (n) & $20.75(77)$ & $33.71(60)$ & 0.002 \\
\hline Serum creatinine concentration [mg/dL], mean (SD) & $0.94(0.32)$ & $1.11(0.4)$ & $<0.001$ \\
\hline eGFR [mL/min*1.73 m²], mean (SD) & $80.85(20.1)$ & $64.88(21.9)$ & $<0.001$ \\
\hline Hemoglobin concentration [g/dL], mean (SD) & $14.05(1.7)$ & $12.93(1.74)$ & $<0.001$ \\
\hline Platelet count $\left[\times 10^{3} / \mathrm{mm}^{3}\right]$, mean (SD) & $217.32(72.17)$ & $228.93(114.6)$ & 0.217 \\
\hline Leukocytes $\left[\times 10^{6} / \mathrm{mm}^{3}\right]$, mean (SD) & $9.09(3.37)$ & $9.53(1.69)$ & 0.188 \\
\hline Total serum cholesterol concentration [mg/dL], mean (SD) & $204.1(50.28)$ & $185.3(52.76)$ & $<0.001$ \\
\hline Serum LDL cholesterol concentration [mg/dL], mean (SD) & $136.22(46.81)$ & $118.45(47.29)$ & $<0.001$ \\
\hline Serum HDL cholesterol concentration [mg/dL], mean, (SD) & $48.86(12.01)$ & $45.2(13.22)$ & 0.002 \\
\hline Serum triglyceride concentration [mg/dL], mean (SD) & $122.57(89.76)$ & $115.6(73.58)$ & 0.34 \\
\hline Serum glucose concentration on admission [mg/dL], mean (SD) & $123.85(41.5)$ & $138.81(52.49)$ & $<0.001$ \\
\hline Potassium concentration [mEq/L], mean (SD) & $4.28(0.41)$ & $4.29(0.49)$ & 0.808 \\
\hline Sodium concentration [mEq/L], mean (SD) & $139.14(2.92)$ & $138.48(4.49)$ & 0.075 \\
\hline Fibrinogen concentration [mg/dL], mean (SD) & $416.43(100.24)$ & $466(115.52)$ & $<0.001$ \\
\hline
\end{tabular}

BMI - body mass index; SBP - systolic blood pressure; sUA - serum uric acid; DBP - diastolic blood pressure; DM2 - diabetes mellitus type 2; CABG - coronary artery bypass grafting; Cx - circumflex artery; EF - ejection fraction; CKD - chronic kidney disease; eGFR - estimated glomerular filtration rate; $\mathrm{HDL}$ - high density lipoprotein; IM - intermediate; LDL - low density lipoprotein; LMCA - left main coronary artery; LAD - left anterior descending artery; M - marginal; PCI - percutaneous coronary intervention; PDA - posterior descending artery; RCA - right coronary artery; SD - standard deviation. 
Table 8. Multivariable logistic regression forward stepwise Wald method - risk of death

\begin{tabular}{|l|c|c|c|}
\multicolumn{1}{|c|}{ Parameter } & p-value & RR & $95 \%$ Cl for RR \\
\hline Age (for every 1 year) & $<0.001$ & 1.086 & $1.065-1.108$ \\
\hline EF (for each 1\% increase) & $<0.001$ & 0.928 & $0.910-0.946$ \\
\hline SUA concentration (for each 1 mg/dL increase) & 0.041 & 1.245 & $1.131-1.370$ \\
\hline Hemoglobin concentration (for each 1 mg/dL increase) & $<0.001$ & 0.685 & $0.611-0.768$ \\
\hline
\end{tabular}

$\mathrm{R}^{2}$ Nagelkerke - 0.72; SUA - serum uric acid; EF - ejection fraction; RR - risk ratio; $95 \% \mathrm{Cl}$ - 95\% confidence interval.

of creatinine and triglycerides as well as a high BMI coexisted with high sUA, as did low HDL cholesterol and EF.

Uric acid is the end result of purine metabolism, which mainly occurs in the liver. Its serum levels depend on many factors, such as purine intake from the diet, liver metabolism, excretion by the kidneys, and intestinal degeneration. ${ }^{14}$ There are several theories about possible pathophysiological mechanisms that are triggered by hyperuricemia. An increased concentration of UA may cause deposits of sodium urate crystals not only in the periarticular tissues but also within the walls of blood vessels. The consequence of the presence of these deposits within the walls of blood vessels is damage to the endothelium, which promotes atherosclerotic changes. The deposits also cause chronic inflammation and damage to the vessel structure, which may all play a role in the occurrence of ACS. ${ }^{15,16}$ An additional mechanism is the influence of high sUA levels on the HMGB1/RAGE signaling pathway. This induces oxidative stress and an inflammatory response, which consequently causes endothelial dysfunction. ${ }^{17}$

Many previous studies have tried to establish the role of sUA in the occurrence of CVD, with contradictory results. In a recent study, researchers from Pakistan reported that in a group of acute myocardial infarction (AMI) patients, sUA levels were higher than in the control group, as was the number of patients with hyperuricemia. ${ }^{18}$

Hyperuricemia has already been linked to the occurrence of other diseases that are known risk factors for ACS, such as hypertension, dyslipidemia, obesity, metabolic syndrome, and CKD., 4 The significance of UA in hypertension is reflected in the guidelines of the European Society of Hypertension, which recommend testing sUA levels as a routine laboratory test in hypertensive patients. ${ }^{19}$ The use of sUA as a marker for cardiac ischemia, which occurs in ACS, might be supported by the fact that adenosine, which is released during ischemia and tissue hypoxia, is degraded by the endothelium to UA, resulting in elevated sUA concentrations. ${ }^{20,21}$

In our study, we observed a positive correlation between the high sUA levels and elevated levels of creatinine and triglycerides. These results overlap with findings from $\mathrm{Ha}-$ jizadeh et al., Tuomilehto et al. and Nagahama et al.8,22,23 A positive correlation was also found for lower EF and lower rates of CKS, as other researchers have also noted. ${ }^{8,24,25}$

Most studies have confirmed the impact of high sUA levels on increased mortality in patients with AMI, especially the STEMI subtype. ${ }^{26,27}$ On the other hand, we can also find contradictory results, ${ }^{8}$ including a study that denies any link between sUA and mortality from all causes, including CVD. ${ }^{28}$

A meta-analysis conducted in 2019 by $\mathrm{He}$ et al. noted that ACS patients with hyperuricemia had a higher risk of all-cause and cardiovascular mortality. ${ }^{29}$ It was also suggested that further studies taking into account the specific subtype of ACS patients are needed. In the present study, almost every third patient with NSTEMI died during the observation. In the group of patients with elevated UA levels, the death rate was 1.5 times higher. A graded increase was demonstrated in the incidence of mortality by increasing quartiles of hyperuricemia.

In addition, Mora-Ramírez et al. showed that high levels of sUA on admission have an impact on short-term mortality among patients admitted due to STEMI with a high prevalence of cardiovascular risk factors. ${ }^{30}$ In 2019, Wei et al. confirmed the influence of UA on long-term mortality in a group of patients with ACS who had undergone PCI. ${ }^{31}$ In our long-term observation, hyperuricemia is associated with higher long-term mortality in patients following NSTEMI. Additional adverse prognostic factors were age, EF and hemoglobin concentration.

These findings encourage consideration of proceeding earlier with UA-lowering treatment in order to reduce cardiovascular risks and potential mortality if they occur, especially since hyperuricemia can be effectively controlled with pharmacological treatment. Prospective studies are necessary to show whether this treatment strategy will bring about the desired results

\section{Limitations}

Our study had several limitations. First, it was a single-center-based study that may not have general application, but the large study group and long follow-up period were certainly strengths. Second, survival data was obtained using personal identification numbers (PESEL) and causes of death were not taken into account - only when death occurred.

\section{Conclusions}

One in 4 patients had sUA levels above the norm. Factors predisposing patients to elevated UA levels were high BMI, CKD, female sex, low HDL, and low left ventricular 
ejection fraction (LVEF). Nearly every $3^{\text {rd }}$ patient with a diagnosis of ACS died during the observation. In the group of patients with elevated UA concentrations, the death rate was 1.5 times higher. Serum UA concentration is an independent risk factor of long-term mortality in patients who have undergone NSTEMI, and is associated with higher in-hospital death rates. Additional adverse prognostic factors were age, low EF and low hemoglobin concentration. Secondary prevention after NSTEMI should entail management of the patients" sUA levels.

\section{ORCID iDs}

Łukasz Kuźma (1) https://orcid.org/0000-0002-4156-5695

Agata Kulikowska (1) https://orcid.org/0000-0003-1038-4418

Anna Kurasz (1) https://orcid.org/0000-0003-0526-3339

Marta Maria Niwińska (1) https://orcid.org/0000-0002-9831-917X

Małgorzata Zalewska-Adamiec (1) https://orcid.org/0000-0002-2466-4093

Sławomir Dobrzycki 이 https://orcid.org/0000-0003-0599-7017

Hanna Bachórzewska-Gajewska

(1) https://orcid.org/0000-0002-0233-6996

\section{References}

1. Roth GA, Johnson C, Abajobir A, et al. Global, regional, and national burden of cardiovascular diseases for 10 causes, 1990 to 2015. J Am Coll Cardiol. 2017;70(1):1-25. doi:10.1016/j.jacc.2017.04.052

2. Anand SS, Islam S, Rosengren A, et al; INTERHEART Investigators. Risk factors for myocardial infarction in women and men: Insights from the INTERHEART study. Eur Heart J. 2008;29(7):932-940. doi:10.1093/ eurheartj/ehn018

3. Li J, Muraki I, Imano H, et al; CIRCS Investigators. Serum uric acid and risk of stroke and its types: The Circulatory Risk in Communities Study (CIRCS). Hypertens Res. 2020;43(4):313-321. doi:10.1038/s41440-0190385-5

4. Yokokawa $H$, Fukuda $H$, Suzuki A, et al. Association between serum uric acid levels/hyperuricemia and hypertension among 85,286 Japanese workers. J Clin Hypertens (Greenwich). 2016;18(1):53-59. doi:10. $1111 /$ jch. 12627

5. Coutinho TA, Turner ST, Peyser PA, Bielak LF, Sheedy $2^{\text {nd }}$ PF, Kullo IJ. Associations of serum uric acid with markers of inflammation, metabolic syndrome and subclinical coronary atherosclerosis. Am J Hypertens. 2007;20(1):83-89. doi:10.1016/j.amjhyper.2006.06.015

6. Rahimi-Sakak F, Maroofi M, Rahmani J, Bellissimo N, Hekmatdoost A. Serum uric acid and risk of cardiovascular mortality: A systematic review and dose-response meta-analysis of cohort studies of over a million participants. BMC Cardiovasc Disord. 2019;19(1):218. doi:10. 1186/s12872-019-1215-z DOI: 10.1186/s12872-019-1215-z

7. Kim SY, Guevara JP, Kim KM, Choi HK, Heitjan DF, Albert DA. Hyperuricemia and coronary heart disease: A systematic review and metaanalysis. Arthritis Care Res (Hoboken). 2010;62(2):170-180. doi:10.1002/ acr.20065

8. Hajizadeh R, Ghaffari S, Salehi R, Mazani S, Aghavali S. Association of serum uric acid level with mortality and morbidity of patients with acute ST-elevation myocardial infarction. J Cardiovasc Thorac Res. 2016;8(2):56-60. doi:10.15171/jcvtr.2016.11

9. Roger VL, Weston SA, Gerber Y, et al. Trends in incidence, severity, and outcome of hospitalized myocardial infarction. Circulation. 2010;121(7):863-869. doi:10.1161/CIRCULATIONAHA.109.897249

10. Car S, Trkulja V. Higher serum uric acid on admission is associated with higher short-term mortality and poorer long-term survival after myocardial infarction: Retrospective prognostic study. Croat Med J. 2009;50(6):559-566. doi:10.3325/cmj.2009.50.559

11. Trkulja V, Car S. On-admission serum uric acid predicts outcomes after acute myocardial infarction: Systematic review and meta-analysis of prognostic studies. Croat Med J. 2012;53(2):162-172. doi:10.3325/ cmj.2012.53.162

12. Çanga $Y$, Emre A, Karataş MB, et al. Prognostic value of serum uric acid levels in patients with non-STEMI undergoing percutaneous coronary intervention. Herz. 2020;45(4):389-396. doi:10.1007/s00059019-04849-3
13. Kuwabara M, Niwa K, Hisatome I, et al. Asymptomatic hyperuricemia without comorbidities predicts cardiometabolic diseases: Five-year Japanese cohort study. Hypertension. 2017;69(6):1036-1044. doi:10. 1161/HYPERTENSIONAHA.116.08998

14. Basseville A, Bates SE. Gout, genetics and ABC transporters. F1000 Biol Rep. 2011;3:23. doi:10.3410/B3-23

15. Dalbeth N, House ME, Aati O, et al. Urate crystal deposition in asymptomatic hyperuricaemia and symptomatic gout: A dual energy CT study. Ann Rheum Dis. 2015;74(5):908-911. doi:10.1136/annrheumdis 2014-206397

16. Kato M, Histome I, Tomikura Y, et al. Status of endothelial dependent vasodilation in patients with hyperuricemia. Am J Cardiol. 2005; 96(11):1576-1578. doi:10.1016/j.amjcard.2005.07.068

17. Cai $W$, Duan $X M$, Liu Y, et al Uric acid induces endothelial dysfunction by activating the HMGB1/RAGE signaling pathway. Biomed Res Int. 2017;2017:4391920. doi:10.1155/2017/4391920

18. Mal K, Jabar AJ, Fatima K, Rizwan A. Incidence of hyperuricemia in patients with acute myocardial infarction: A case-control study. Cureus. 2020;12(1):e6722. doi:10.7759/cureus.6722

19. European Society of Hypertension-European Society of Cardiology Guidelines Committee. 2003 European Society of HypertensionEuropean Society of Cardiology guidelines for the management of arterial hypertension. J Hypertens. 2003;21(6):1011-1053. doi:10. 1097/00004872-200306000-00001

20. Raatikainen MJ, Peuhkurinen KJ, Hassinen IE. Contribution of endothelium and cardiomyocytes to hypoxia-induced adenosine release. J Mol Cell Cardiol. 1994;26(8):1069-1080. doi:10.1006/jmcc.1994.1127

21. Kroll K, Bukowski TR, Schwartz LM, Knoepfler D, Bassingthwaighte JB. Capillary endothelial transport of uric acid in guinea pig heart. Am J Physiol. 1992;262(2 Pt 2):H420-H431. doi:10.1152/ajpheart.1992. 262.2. $\mathrm{H} 420$

22. Tuomilehto J, Zimmet $\mathrm{P}$, Wolf E, Taylor R, Ram P, King H. Plasma uric acid level and its association with diabetes mellitus and some biologic parameters in a biracial population of Fiji. Am J Epidemiol. 1988; 127(2):321-336. doi:10.1093/oxfordjournals.aje.a114807

23. Nagahama K, Iseki K, Inoue T, Touma T, Ikemiya Y, Takishita S. Hyperuricemia and cardiovascular risk factor clustering in a screened cohort in Okinawa, Japan. Hypertens Res. 2004;27(4):227-233. doi:10. 1291/hypres.27.227

24. Ranjith N, Myeni NN, Sartorius B, Mayise C. Association between hyperuricemia and major adverse cardiac events in patients with acute myocardial infarction. Metab Syndr Relat Disord. 2017;15(1):18-25. doi:10.1089/met.2016.0032

25. Eleftheriadis T, Golphinopoulos S, Pissas G, Stefanidis I. Asymptomatic hyperuricemia and chronic kidney disease: Narrative review of a treatment controversial. J Adv Res. 2017;8(5):555-560. doi:10. 1016/j.jare.2017.05.001

26. Xu Q, Zhang M, Abeysekera IR, Wang X. High serum uric acid levels may increase mortality and major adverse cardiovascular events in patients with acute myocardial infarction. Saudi Med J. 2017;38(6): 577-585. doi:10.15537/smj.2017.6.17190

27. Celik T, lyisoy A. Uric acid levels for the prediction of prognosis in patients with acute ST elevation myocardial infarction: A new potential biomarker. Int J Cardiol. 2010;145(1):e21-e22. doi:10.1016/j.ijcard. 2008.12.073

28. Sakata K, Hashimoto T, Ueshima H, Okayama A; NIPPON DATA 80 Research Group. Absence of an association between serum uric acid and mortality from cardiovascular disease: NIPPON DATA 80, 1980-1994. National Integrated Projects for Prospective Observation of Non-communicable Diseases and its Trend in the Aged. Eur J Epidemiol. 2001;17(5):461-468. doi:10.1023/a:1013735717961

29. He C, Lin P, Liu W, Fang K. Prognostic value of hyperuricemia in patients with acute coronary syndrome: A meta-analysis. Eur J Clin Invest. 2019;49(4):e13074. doi:10.1111/eci.13074

30. Mora-Ramírez M, Estevez-Garcia IO, Irigoyen-Camacho ME, Bojalil R, Gonzalez-Pacheco H, Amezcua-Guerra LM. Hyperuricemia on admission predicts short-term mortality due to myocardial infarction in a population with high prevalence of cardiovascular risk factors. Rev Invest Clin. 2017;69(5):247-253. doi:10.24875/ric.17002167

31. Wei G, Dahao Y, Dengxuan W, et al. Hyperuricemia and long-term mortality in patients with acute myocardial infarction undergoing percutaneous coronary intervention. Ann Trans/ Med. 2019;7(22):636. doi:10.21037/atm.2019.10.110 